\title{
Magnetic field evolution in dwarf and Magellanic-type galaxies
}

\author{
H. Siejkowski ${ }^{1}$, M. Soida ${ }^{2}$, and K. T. Chyży ${ }^{2}$ \\ ${ }^{1}$ AGH University of Science and Technology, ACC Cyfronet AGH, ul. Nawojki 11, PO Box 386, 30-950, Kraków 23, Poland \\ e-mail: h.siejkowski@cyfronet.pl \\ 2 Astronomical Observatory of the Jagiellonian University, ul. Orla 171, 30-244 Kraków, Poland
}

Received 6 February 2017 / Accepted 2 December 2017

\begin{abstract}
Aims. Low-mass galaxies radio observations show in many cases surprisingly high levels of magnetic field. The mass and kinematics of such objects do not favour the development of effective large-scale dynamo action. We attempted to check if the cosmic-ray-driven dynamo can be responsible for measured magnetization in this class of poorly investigated objects. We investigated how starburst events on the whole, as well as when part of the galactic disk, influence the magnetic field evolution.

Methods. We created a model of a dwarf/Magellanic-type galaxy described by gravitational potential constituted from two components: the stars and the dark-matter halo. The model is evolved by solving a three-dimensional (3D) magnetohydrodynamic equation with an additional cosmic-ray component, which is approximated as a fluid. The turbulence is generated in the system via supernova explosions manifested by the injection of cosmic-rays.

Results. The cosmic-ray-driven dynamo works efficiently enough to amplify the magnetic field even in low-mass dwarf/Magellanictype galaxies. The $e$-folding times of magnetic energy growth are 0.50 and $0.25 \mathrm{Gyr}$ for the slow $\left(50 \mathrm{~km} \mathrm{~s}^{-1}\right)$ and fast $\left(100 \mathrm{~km} \mathrm{~s}^{-1}\right)$ rotators, respectively. The amplification is being suppressed as the system reaches the equipartition level between kinetic, magnetic, and cosmic-ray energies. An episode of star formation burst amplifies the magnetic field but only for a short time while increased star formation activity holds. We find that a substantial amount of gas is expelled from the galactic disk, and that the starburst events increase the efficiency of this process.
\end{abstract}

Key words. dynamo - galaxies: dwarf - galaxies: magnetic fields - galaxies: starburst - magnetohydrodynamics (MHD) methods: numerical

\section{Introduction}

Dwarf galaxies are the most abundant galaxies in the Universe but they are also difficult to observe due to their low luminosity and small size. Evolution of stars and the interstellar medium (ISM) in dwarfs can be studied in the absence of spiral density waves and on a small scale. Low masses and metallicities of dwarf galaxies suggest that they could have been the building blocks of larger galaxies, constituting the key element in the theory of hierarchical galaxy formation.

There is no clear break to distinguish a dwarf from a larger (irregular) galaxy of the Magellanic type but according to working definitions, all galaxies fainter than $M_{v}=-17$ are regarded as dwarfs (Tolstoy et al. 2009). The Small Magellanic Cloud is close to this boundary and resembles some larger dwarfs from the Local Group, such as IC 1613 and NGC 6822, while the Large Magellanic Cloud is more like low-luminosity spirals; for example, M33.

In massive spiral galaxies, the ISM involves sufficiently strong shearing motions due to differential rotation and the Coriolis force to generate large-scale magnetic fields by dynamo processes (Widrow 2002). Such magnetic fields have been discovered in a number of spiral galaxies and investigated through synchrotron emission and Faraday rotation effects (Beck 2016). The low-mass, dwarf/irregular galaxies are also rich in gas but reveal much more chaotic gas motions and slow rotation, which might not provide favourable conditions for generating strong and large-scale magnetic fields.

In spite of these considerations, strong magnetic fields ( $>10 \mu \mathrm{G})$ were actually observed in the dwarf galaxies IC 10
(Chyży et al. 2016) and NGC 1569 (Kepley et al. 2010), as well as even a regular field in NGC 4449 (Chyży et al. 2000). However, a systematic survey of galaxies in the Local Group (Chyży et al. 2011) led to radio detection at $2.64 \mathrm{GHz}$ of only 3 out of 12 dwarfs. The results obtained indicated that the mean strength of magnetic fields in the local dwarf galaxies is small, about $4 \mu \mathrm{G}$, and related to the galactic star formation rate (SFR).

The generation and evolution of magnetic fields in dwarf galaxies were simulated by a cosmic-ray (CR)-driven dynamo in the shearing-box approximation (Siejkowski et al. 2010) as well as in the global model (Siejkowski et al. 2014). In this model, the CRs and magnetic seed fields are injected into the ISM by randomly exploding supernovae $(\mathrm{SN})$. The simulations prove that even in the slowly rotating dwarf galaxies this kind of dynamo is able to amplify magnetic field with an exponential growth rate, reproducing the observed magnetic field strengths. The $e$-folding time of magnetic azimuthal flux growth appeared to be correlated with the initial rotation speed and was rather long (about $1 \mathrm{Gyr}$ ) for a galaxy rotating with a speed $v_{\phi}=40 \mathrm{~km} \mathrm{~s}^{-1}$. The simulations also show a significant loss of magnetic field from the simulated domain (Siejkowski et al. 2010). Such an effect depends on the $\mathrm{SN}$ rate and is due to gas escaping through galactic winds driven by cosmic rays (see also Hanasz et al. 2013).

In these simulations, the production of stars was assumed to be the same throughout the evolution of galaxies. However, this simplification may be too strong. Investigation of star formation history (SFH) of dwarf galaxies indicates that in fact their rates of star formation are quite diverse and show fluctuations in time (Weisz et al. 2011). According to numerical simulations, 
the tidal-driven changes in star formation are rather short-lived, with a typical duration of the activity of about $10^{8} \mathrm{yr}$ (Di Matteo et al. 2008). Strong starbursts during which the star formation rate is increased by a factor larger than 5 are rare. IC 1613 is a good example of a dwarf that calmly produces stars over its entire lifetime, while showing also some fluctuations (Skillman et al. 2014). The galaxy has a small Hi mass of $8 \times 10^{7}$ and a stellar mass of $1.1 \times 10^{8}$ (Woo et al. 2008). The current SFR is $0.003 M_{\odot} \mathrm{yr}^{-1}$ (Mateo 1998), but the $\mathrm{SFH}$ reveals some episodes in the galaxy life with the SFR enhancement of about threefold (Skillman et al. 2014).

Another aspect of evolution of low-mass galaxies not addressed in numerical experiments to date is the often observed irregular distribution of star-forming regions in galactic disks. Such an asymmetric distribution is evident in for example IC 10 , dominated by a giant HII region in its southern part. The observed morphology and the strength of magnetic field are strongly related to the distribution of HII regions within the galactic disk (Chyży et al. 2016).

So as to address the above issues, in this paper a series of 3D simulations of the CR-driven dynamo for low-mass galaxies are performed. In the first place, we simulate a calm evolution of galaxies at different speeds of rotation and different (but constant in time) levels of star formation activity. Below, we introduce a starburst (a rapid enhancement of star formation) and study its impact on magnetic field, while finally investigating how a localised starburst, restricted to just part of the galactic disk, changes the magnetic field topology, and how this is related to the topology for the global starburst.

\section{Numerical model and setup}

\subsection{Cosmic-ray-driven dynamo}

The CR-driven dynamo model in dwarf/irregular galaxies has been implemented into a numerical code called GoDUNOVMHD (Kowal et al. 2009). The code solves the system of resistive magnetohydrodynamic (MHD) equations in a conservative form in 3D space (e.g. Goedbloed \& Poedts 2004):

$$
\frac{\partial \rho}{\partial t}+\nabla \cdot(\rho \boldsymbol{v})=0
$$

$\frac{\partial \rho \boldsymbol{v}}{\partial t}+\nabla\left[\rho \boldsymbol{v} \boldsymbol{v}+\left(p+p_{\mathrm{cr}}+\frac{B^{2}}{8 \pi}\right) \boldsymbol{I}-\frac{\boldsymbol{B} \boldsymbol{B}}{4 \pi}\right]=-\rho \nabla \phi$

$\frac{\partial \boldsymbol{A}}{\partial t}-\boldsymbol{v} \times(\nabla \times \boldsymbol{A})-\eta \nabla \times(\nabla \times \boldsymbol{A})=0$,

where $\rho, p$, and $\boldsymbol{v}$ are the gas density, pressure, and velocity, respectively, $p_{\mathrm{cr}}$ is the cosmic-ray pressure, $\boldsymbol{A}$ is the vector potential, $\boldsymbol{B} \equiv \nabla \times \boldsymbol{A}$ is the magnetic field, $\phi$ is the gravitational potential, $\eta$ is the magnetic turbulent diffusivity and $\boldsymbol{I}$ is the identity matrix. To close the system an isothermal equation of state is assumed:

$p \equiv \frac{c_{s}^{2}}{\gamma} \rho$,

where $c_{s}$ is the isothermal speed of sound and $\gamma$ is the adiabatic index of the gas.
Cosmic rays form a key component of the model. In our case they are approximated as a fluid, whose evolution is described by the diffusion-advection transport equation following (Schlickeiser \& Lerche 1985):

$\frac{\partial e_{\mathrm{cr}}}{\partial t}+\nabla\left(e_{\mathrm{cr}} \boldsymbol{v}\right)=\nabla\left(\hat{K} \nabla e_{\mathrm{cr}}\right)-p_{\mathrm{cr}}(\nabla \cdot \boldsymbol{v})+Q$,

where $e_{\mathrm{cr}}$ is the CR energy density, $\hat{K}$ is the diffusion tensor, $\boldsymbol{v}$ is the plasma velocity, $p_{\text {cr }}$ is the CR pressure, and $Q$ is the source term of $e_{\mathrm{cr}}$. CR pressure is related to the $e_{\mathrm{cr}}$ via the adiabatic CR index:

$p_{\mathrm{cr}} \equiv\left(\gamma_{\mathrm{cr}}-1\right) e_{\mathrm{cr}}$.

The diffusion of CRs is anisotropic (Giacalone \& Jokipii 1999) with respect to the magnetic field, hence the diffusion tensor $\hat{K}$ from Eq. (5) is defined as follows (Ryu et al. 2003):

$K_{i j}=K_{\perp} \delta_{i j}+\left(K_{\|}-K_{\perp}\right) n_{i} n_{j}$,

where $K_{\perp}$ and $K_{\|}$are the diffusion coefficients perpendicular and parallel to the local magnetic field, respectively, and $n_{i} \equiv B_{i} / B$ is the $i$ th component of the vector tangent to the local magnetic field.

In the CR-driven dynamo model the source term $Q$ in Eq. (5) is attributed to the SN remnants (Hanasz et al. 2004) in which CR particles are accelerated. A fraction of the kinetic energy output of the SN explosion is converted into the acceleration of the CRs in the shock front. The conversion rate is $10-30 \%$ (Dorfi 2000) of the kinetic energy. We assume that the kinetic energy output of the SN explosion is $10^{51} \mathrm{erg}$ and the conversion rate is $10 \%$. Each SN explosion is modelled by a 3D Gaussian distribution of CR energy input and is added to the source term $Q$ in Eq. (5). Some of the explosions are magnetized and besides the input of $\mathrm{CR}$ energy, a randomly oriented dipole magnetic field is injected into the ISM. The magnetized SN explosions occurs only for the first $t_{\text {mag }}$ time and only $10 \%$ of all explosions in that time are magnetized. This is to seed the dynamo action.

The position of each SN explosion is chosen randomly according to the local gas density. Schmidt (1959) and Kennicutt (1989) showed that in a simple self-gravitational picture the large-scale SFR volume density $\rho_{\mathrm{SRF}}$ scales with gas density as $\rho_{\mathrm{SFR}} \propto \rho^{3 / 2}$. Using this relation we can build a cumulative distribution function of SN explosions in a galactic disk. Assuming a constant $\mathrm{SN}$ explosion frequency, $f_{\mathrm{SN}}$, we can calculate a number of exploding stars and find their positions according to a cumulative distribution function in each time step. The $f_{\mathrm{SN}}$ is modulated in time with a period of $200 \mathrm{Myr}$, in which for the first $40 \mathrm{Myr}$ the $\mathrm{SNe}$ are active, before being set to zero; after the following $160 \mathrm{Myr}$ the process is repeated. The $f_{\mathrm{SN}}$ value represents the SN explosion frequency during the active interval, so to compute the mean it should be multiplied by $40 \mathrm{Myr} / 200 \mathrm{Myr}=0.2$. The period $200 \mathrm{Myr}$ has been found by Siejkowski et al. (2010) as the most effective for the amplification of the magnetic field in dwarf galaxies. Additionally they found that the longer the period of silence in SN activity the more effective the amplification.

The dwarf/irregular galaxy gravitational potential well is given by two components: dark matter (DM) halo and thin stellar disc. This type of galaxy does not have bulge (Governato et al. 2010) which is usually present in bigger objects, like granddesign spirals. The stars are distributed in infinitesimally thin Kuzmin's disk which generates a potential well of the form (e.g. Binney \& Tremaine 2008):

$\phi_{*}(R, z)=-\frac{G M_{*}}{\sqrt{R^{2}+(a+|z|)^{2}}}$, 
Table 1. Parameters of the reference models.

\begin{tabular}{llll}
\hline \hline Model name & v50 & v100 & Unit \\
\hline Parameter & & & \\
\hline$M_{*}$ & 2.4 & 15 & $10^{9} M_{\odot}$ \\
$\rho_{0}$ & 10.8 & 7.0 & $10^{6} M_{\odot} \mathrm{kpc}^{-3}$ \\
$r_{0}$ & 1.8 & 2.2 & $\mathrm{kpc}$ \\
$r_{g}$ & 29.5 & 29.5 & $10^{6} M_{\odot} \mathrm{kpc}^{-3}$ \\
$R_{c}$ & 1.0 & 2.0 & $\mathrm{kpc}^{-2} \mathrm{Gyr}^{-1}$ \\
$f_{\mathrm{SN}}$ & $3 \times 10^{3}$ & $1 \times 10^{3}$ & $\mathrm{kpc}^{-2} \mathrm{kpc}$ \\
Galaxy radius & 4.5 & 7.5 & $\mathrm{kpc}$ \\
Grid size & 47 & 78 & $\mathrm{pc}$ \\
\hline Rotation speed & 50 & 100 & $\mathrm{~km} \mathrm{~s}^{-1}$ \\
\hline
\end{tabular}

where $G$ is the gravitational constant, $M_{*}$ is the total mass of stars, $a$ is the radial scalelength, and $R$ is the radius in equatorial plane $(z=0)$, that is, $R \equiv \sqrt{x^{2}+y^{2}}$.

The DM halo is approximated by a "modified isothermal sphere" which belongs to a class of double (broken) power-law density distributions (see Mo et al. 2010). The density profile is given by $\rho_{\mathrm{h}}^{\text {iso }}(r)=\rho_{0} /\left[1+\left(r / r_{0}\right)^{2}\right]$, and the potential is described as:

$\phi_{\mathrm{h}}^{\mathrm{iso}}(r)=4 \pi G \rho_{0} r_{0}^{2}\left[\frac{1}{2} \ln \left(1+x^{2}\right)+\frac{\arctan x}{x}\right]$,

where $\rho_{0}$ is the central density, $r_{0}$ is the core radius and $r$ is the distance to the centre $r \equiv \sqrt{R^{2}+z^{2}}$ and $x \equiv r / r_{0}$.

The initial condition of the galaxy model is set to hydrostatic equilibrium. The gas density distribution in the equatorial plane is assumed to have following form:

$\rho(R, z=0)=\frac{\rho_{g}}{\left[1+\left(\frac{R}{R_{c}}\right)^{2}\right]^{2}}$,

where $\rho_{g}$ and $R_{C}$ are the central gas density and core radius, respectively. Then to find the global gas distribution we use the "potential method" following Wang et al. (2010). From the gas distribution the $\mathrm{CR}$ component distribution is found assuming that they are in pressure equilibrium, namely $\beta \equiv p_{\mathrm{CR}} / p_{\text {gas }}=1$. The magnetic field in $t=0$ is set to zero $\left(\alpha \equiv p_{\text {mag }} / p_{\text {gas }}=0\right)$.

\subsection{Model description}

We setup the gas temperature to about $6000 \mathrm{~K}$, which corresponds to $c_{s}=7 \mathrm{~km} \mathrm{~s}^{-1}$. The magnetic diffusivity is set to $3 \times 10^{25} \mathrm{~cm}^{2} \mathrm{~s}^{-1}$ following Hanasz et al. (2009a), who have shown that this value is optimal for the growth of the magnetic field in the buoyancy-driven dynamo. Following Ryu et al. (2003) the CR adiabatic index was set to $\gamma_{\mathrm{cr}}=14 / 9$, and the CR diffusion coefficients are following: $K_{\perp}=3 \times 10^{26} \mathrm{~cm}^{2} \mathrm{~s}^{-1}$ and $K_{\|}=3 \times 10^{27} \mathrm{~cm}^{2} \mathrm{~s}^{-1}$. Strong et al. (2007) argues that the typical value for CR diffusion coefficient is about $(3 / 5) \times 10^{28} \mathrm{~cm}^{2} \mathrm{~s}^{-1}$, however this highly impacts the time-step value and makes the simulation unfeasible. This issue has been investigated by Hanasz et al. (2009a), who have shown that the magnetic field growth only slightly depends on the $K_{\|}$value, but the key factor is the anisotropy of the CR diffusion that we apply. The 3D space domain is represented as a grid of $256 \times 256 \times 128$ points in $x$, $y$, and $z$ direction, respectively. We set the boundary condition to outflow in all directions.
Table 2. The applied $f_{\mathrm{SN}}$ in $10^{3} \mathrm{kpc}^{-2} \mathrm{Gyr}^{-1}$ for our models.

\begin{tabular}{lccc}
\hline \hline Model & Base level & Starburst event & Region \\
\hline v50 & 3.0 & - & - \\
v50x1Q & 3.0 & 3.0 & Quarter \\
v50x2 & 3.0 & 6.0 & Whole disk \\
v50x2Q & 3.0 & 6.0 & Quarter \\
v50x5 & 3.0 & 15.0 & Whole disk \\
v50x5Q & 3.0 & 15.0 & Quarter \\
\hline v100 & 1.0 & - & - \\
v100x1Q & 1.0 & 1.0 & Quarter \\
v100x2 & 1.0 & 2.0 & Whole disk \\
v100x2Q & 1.0 & 2.0 & Quarter \\
\hline
\end{tabular}

Notes. The base level is used throughout the whole simulation, except if there is a starburst event, where the SFR is enhanced. The region describes whether the starburst event is in the whole disk or localized only to one quarter of the galaxy.

We define two reference models with rotational velocities of approximately 50 and $100 \mathrm{~km} \mathrm{~s}^{-1}$. The parameters of the models are given in Table 1 . We set $f_{\mathrm{SN}}$ to $3 \times 10^{3} \mathrm{kpc}^{-2} \mathrm{Gyr}^{-1}$ for the slower rotation model, and three times lower for the faster rotation model. For both models such values give similar global $\mathrm{SFR} \approx 0.025 M_{\odot} \mathrm{yr}^{-1}$ in the ongoing phase of the SN activity (Sect. 2.1) and a mean value of the global SFR of about $0.005 M_{\odot} \mathrm{yr}^{-1}$ during the $200 \mathrm{Myr}$ activity period. These values of parameters also provide stable code execution during the starburst phase of galaxy evolution. The corresponding actual galaxies can be found among non-starbursting dwarf galaxies (Chyży et al. 2011); for example IC 1613 with a global SFR of $0.003 M_{\odot} \mathrm{yr}^{-1}$.

To study the influence of the starburst events on the magnetic field evolution in the galaxy we introduce such events into the reference models. This is modelled by a single event (one period of SN modulation) during which we inject $k \times f_{\mathrm{SN}}$. For model v50 this event occurs at $t=6 \mathrm{Gyr}$ and we investigate $k=2,5$ and for model $\mathrm{v} 100$ at $t=4 \mathrm{Gyr}$ and we investigate $k=2$. The event lasts for $100 \mathrm{Myr}$ during the $200 \mathrm{Myr}$ period, and the mean $\mathrm{SN}$ activity is $0.5 k \times f_{\mathrm{SN}}$. Additionally we modify this event to inject the $\mathrm{SNe}$ only in one quarter of the galaxy, to study the localised burst events. We note that the effective $f_{\mathrm{SN}}$ value in the case of a localised event is four times stronger in the quarter. The total number of SN explosions is the same for the reference models and the localised ones. The base levels in the models and the starburst events are shown in Table 2.

Throughout the paper we use the following notation for the model names: " $v$ " and a number stands for the rotational velocity, " $x$ " and a number denotes the burst event and the number corresponds to the $k$ value, "Q" marks models in which the event occurs only in a quarter of the galaxy disc. For example, the name v50x2Q represents a model of a galaxy with a rotation as fast as $50 \mathrm{~km} \mathrm{~s}^{-1}$, in which the burst event is two times the base $f_{\mathrm{SN}}$ value and the $\mathrm{SNe}$ has been injected only in a quarter of the galactic disc.

\section{Results}

The evolution of the total magnetic energy, $E_{\text {mag }}$, and mean magnetic azimuthal component, $\left\langle B_{\phi}\right\rangle$, are shown in Figs. 1 and 2, respectively. To estimate the $\left\langle B_{\phi}\right\rangle$ we calculate the $B_{\phi}^{i, j, k}$ in each grid point and then we sum them all and divide by the number 

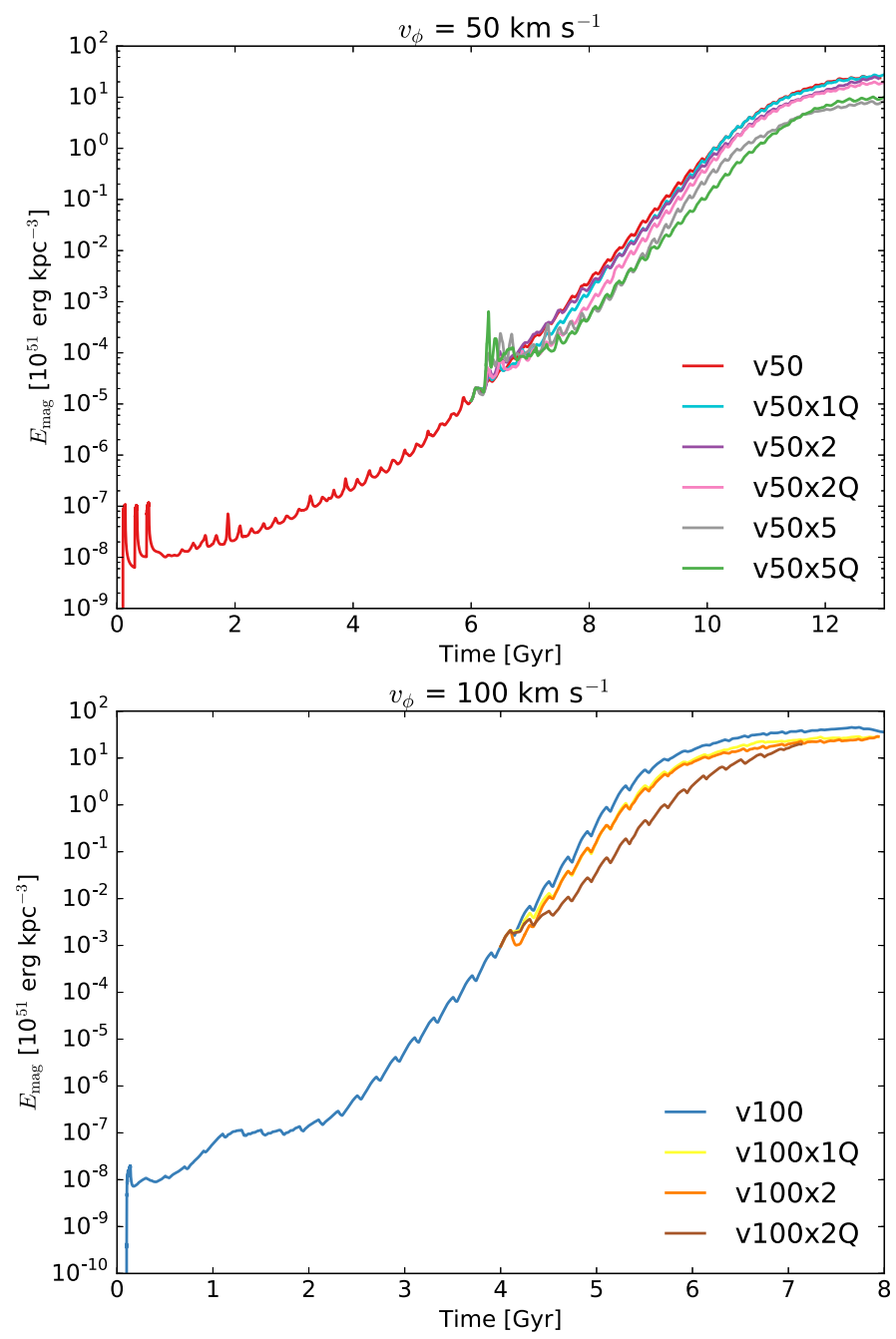

Fig. 1. Evolution of the total magnetic energy density.

of grid points. We restrict the summing of the grid points to $|z| \leq 1 \mathrm{kpc}$.

All the models investigated show an exponential growth of the magnetic field in time. In the case of models with $v_{\phi}^{\max }=50 \mathrm{~km} \mathrm{~s}^{-1}$ in the beginning of the simulation we inject three events of magnetized SN explosions (Fig. 1). This is visible in the magnetic energy plot as three peaks in the first $500 \mathrm{Myr}$. Then the magnetic field injection is stopped, and the growth is caused only by the dynamo operation. We found that for models with $v_{\phi}^{\max }=100 \mathrm{~km} \mathrm{~s}^{-1}$ only one such event is enough to seed the dynamo.

The models with $v_{\phi}^{\max }=50 \mathrm{~km} \mathrm{~s}^{-1}$ show constant growth of the magnetic field energy, just after the injection of magnetized $\mathrm{SNe}$ is stopped. The $e$-folding time is about $0.5 \mathrm{Gyr}$ for the magnetic energy (see Table 3) and the saturation point is reached after 12 Gyr. The azimuthal component evolution shows two phases: the first, the slow one which occurs between $t=1 \mathrm{Gyr}$ and $4 \mathrm{Gyr}$, and the second, the fast one after $t=4 \mathrm{Gyr}$ which lasts until the saturation point around $t=12 \mathrm{Gyr}$. The mean $e$-folding time for $\left\langle B_{\phi}\right\rangle$ is $0.91 \mathrm{Gyr}$.

The faster rotators, after first injection of the magnetic field, start to amplify the magnetic field energy exponentially and around $t=1$ Gyr the growth stops for the next 1 Gyr. After that the dynamo restarts, and the amplification is exponential with $e$-folding as high as $0.25 \mathrm{Gyr}$ (see Table 3). The saturation is reached around $t=5.5 \mathrm{Gyr}$. The mean azimuthal component of
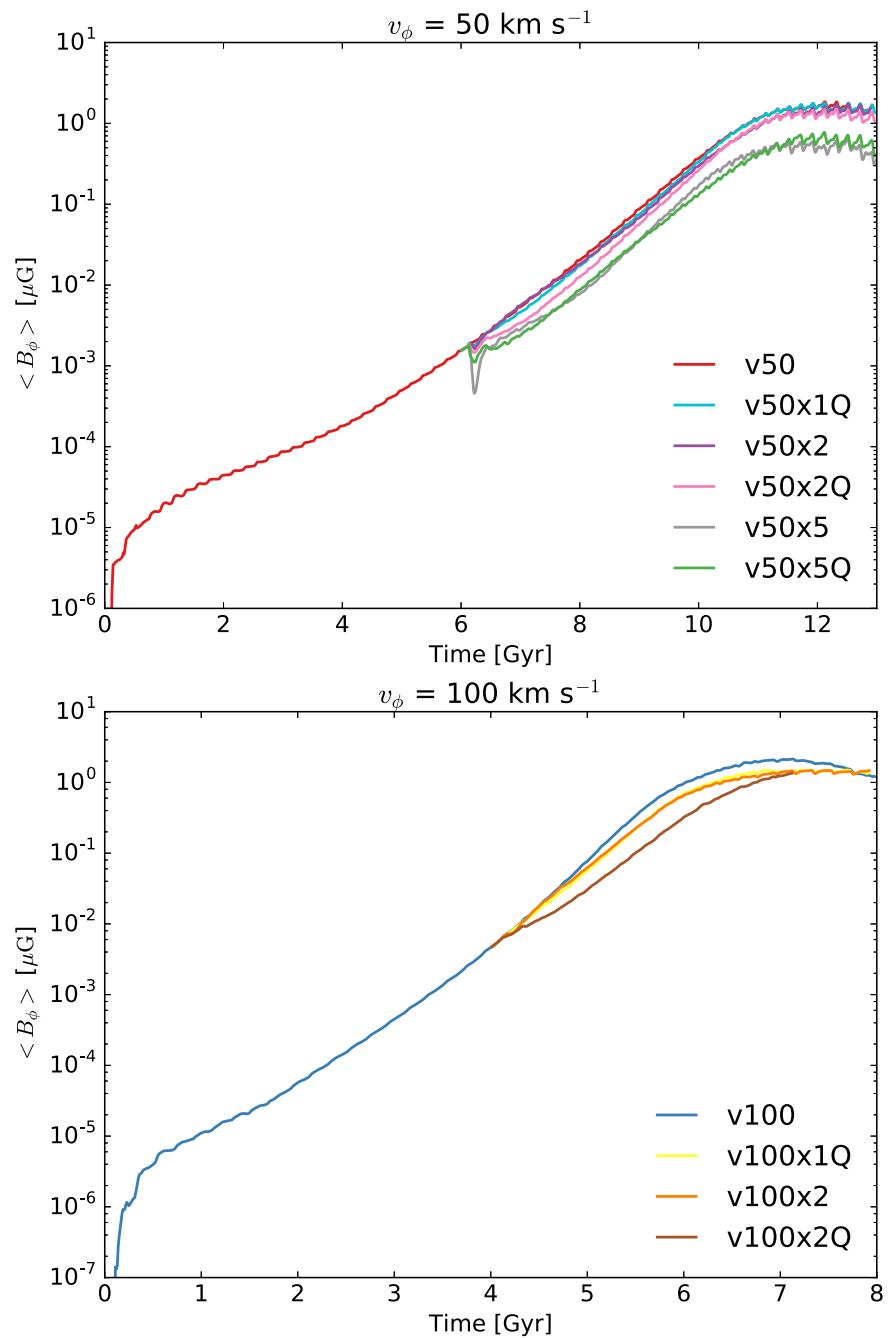

Fig. 2. Evolution of the mean azimuthal magnetic component. To use the logarithmic scale for the azimuthal component plot the absolute value is plotted; if the flux starts rapidly dropping to very low values and then rapidly grows, this is a signature of sign turnover.

the magnetic field is amplified exponentially with the $e$-folding time equal to $0.44 \mathrm{Gyr}$.

We show the $e$-folding times of magnetic field evolution for all the models investigated in Table 3 . Since the burst episodes influence the magnetic evolution we report the global mean values for $1 \mathrm{Gyr}<t<11 \mathrm{Gyr}$ and $0.5 \mathrm{Gyr}<t<5.5 \mathrm{Gyr}$ for v50 and v100, respectively. For all models representing galaxies with rotation of about $50 \mathrm{~km} \mathrm{~s}^{-1}$ we obtain the $e$-folding times between $8 \mathrm{Gyr}<t<11 \mathrm{Gyr}$, and for $100 \mathrm{~km} \mathrm{~s}^{-1}$ between $4.5 \mathrm{Gyr}<t<5.5 \mathrm{Gyr}$.

\subsection{Energy balance}

In our models of galaxies there are three components which contribute to the total energy of the system, namely thermal gas, CRs, and magnetic fields. To show the energy evolution for each component, the following ratios are calculated:

$\alpha_{E} \equiv E_{\mathrm{mag}} / E_{\mathrm{trub}} \quad$ and $\quad \beta_{E} \equiv E_{\mathrm{CR}} / E_{\mathrm{trub}}$.

The turbulence energy, $E_{\text {turb }}$ is estimated by:

$E_{\mathrm{turb}}=\frac{1}{2} M\left(\operatorname{Var}\left(v_{r}\right)+\operatorname{Var}\left(v_{\phi}\right)+\operatorname{Var}\left(v_{z}\right)\right)$, 


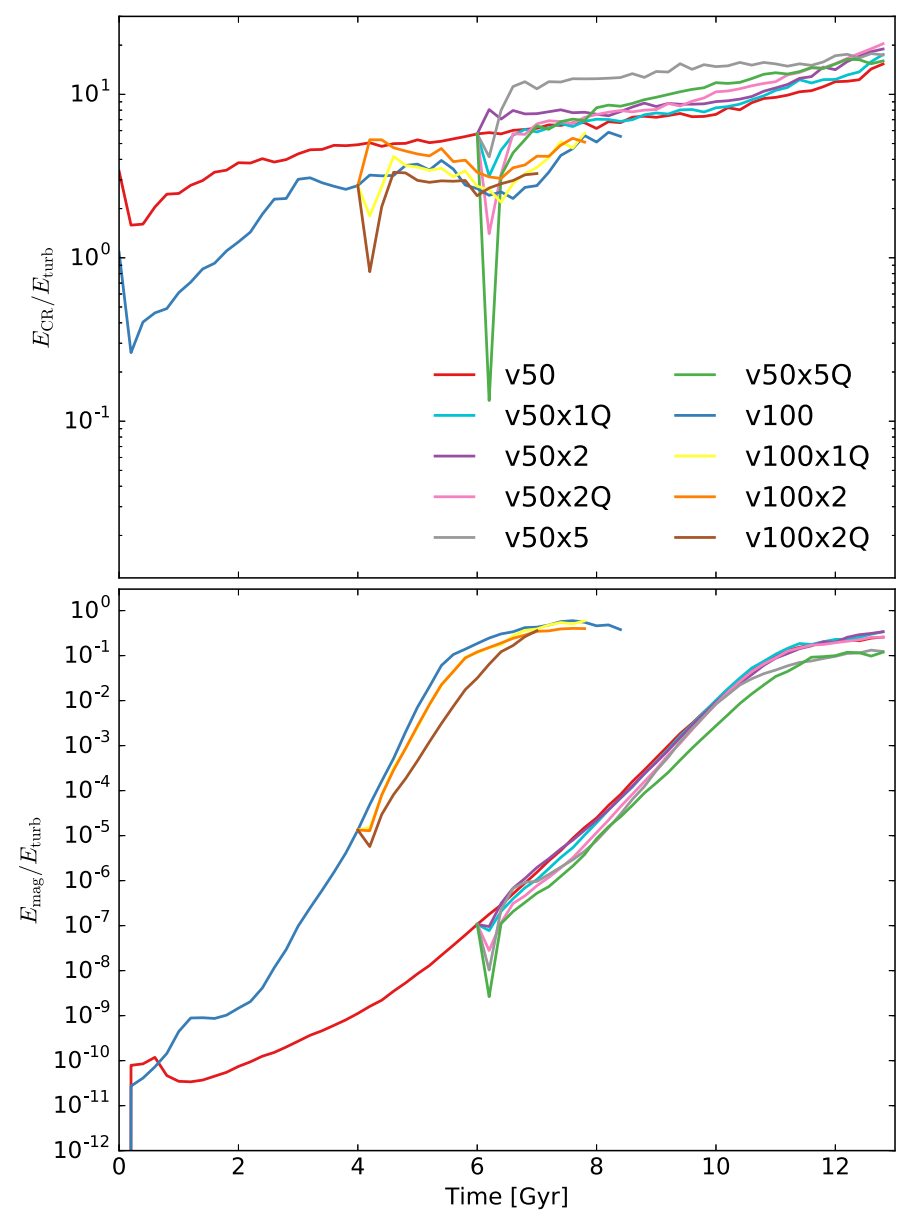

Fig. 3. Evolution of the energy ratios. The upper panel shows $\alpha_{E}$ and the lower one $\beta_{E}$.

where $v_{r}, v_{\phi}$, and $v_{z}$ are the velocity vector components expressed in aziumthal coordinates, $M$ is the total gas mass in the domain, and $\operatorname{Var}(X)$ is the variance, that is, $\operatorname{Var}(X)=\sum_{i=1}^{n}\left(x_{i}-\mu\right)^{2} / n$, where $\mu=\sum_{i=1}^{n} x_{i} / n$.

The evolution of $\alpha_{E}$ and $\beta_{E}$ for the reference models are shown in Fig. 3. We restrict the computations of $\alpha_{E}$ and $\beta_{E}$ to the disk region, that is, $|z|<1 \mathrm{kpc}$. The energy density of the CRs during the evolution is slowly increasing because of the $\mathrm{SNe}$ injection. The final value of $\beta_{E}$ is between 3.0 and 20.0 for each of the investigated models. The evolution of $\alpha_{E}$ ratio, which is crucial for the dynamo action is shown as well. The initial value, at $t=0$ is set to 0 , and after the simulation starts, the magnetic field is seeded by the magnetized SN explosions. The energy ratio $\alpha_{E}$ after first injections is between $10^{-11}$ and $10^{-10}$ and depends on the galaxy size. The exponential growth is then visible until the saturation around $\alpha_{E} \simeq 0.5$.

The issue of the energy balance in the system between turbulent kinetic energy, CRs, and magnetic fields has been investigated by Hanasz et al. (2009a) in the local simulations of $\mathrm{CR}$-driven dynamo in massive galaxies. They found that the evolution of $E_{\mathrm{CR}} / E_{\text {trub }}$ depends on the CR diffusion coefficients and if the latter is higher, the ratio after the simulation is lower. In our work the adopted value of the CR diffusion is $10 \%$ of the realistic values because of the time step limitations. The explicit algorithm of the CR diffusion causes the time step to be significantly short when realistic values of diffusion coefficients are used. The simulation results show that in the case of global dwarf galaxy simulation the CR energy is comparable to the turbulent
Table 3. $e$-Folding times given in Gyr for the evolution of the magnetic energy (1) and azimuthal component of the magnetic field (2).

\begin{tabular}{lcc}
\hline \hline Model & $(1)$ & $(2)$ \\
\hline v50* & 0.50 & 0.91 \\
v50 & 0.38 & 0.75 \\
v50x1Q & 0.36 & 0.71 \\
v50x2 & 0.38 & 0.74 \\
v50x2Q & 0.35 & 0.70 \\
v50x5 & 0.36 & 0.74 \\
v50x5Q & 0.38 & 0.77 \\
\hline v100* & 0.25 & 0.44 \\
v100 & 0.18 & 0.34 \\
v100x1Q & 0.19 & 0.38 \\
v100x2 & 0.19 & 0.40 \\
v100x2Q & 0.23 & 0.46 \\
\hline
\end{tabular}

Notes. The models denoted with a star show the global mean values, as defined in the text.

kinetic energy. Hanasz et al. (2009a) and Snodin et al. (2006) find excess CR energy in the numerical simulations of galaxy evolution and $\mathrm{CR}$ gas. It also seems that the ratio of $\mathrm{CR}$ to other forms of energy in the ISM is not yet well constrained by the observations (Strong et al. 2007; Stepanov et al. 2014).

\section{Discussion and conclusions}

Our models represent dwarf and Magellanic-type irregulars. Both reference models (v50 and v100) correspond to a calm phase of galactic evolution. Our mean surface density of the SFR $\left(f_{\mathrm{SN}}\right)$ covers the lower end of surface star formation rate (SSFR). The objects IC 1613 and IC 2574 may represent good real examples (see e.g. Fig. 5 in Jurusik et al. 2014). Our results show that a faster rotator amplifies the magnetic field much faster, regardless of the lower star formation activity applied. Stronger action of the dynamo for a faster rotating galaxy was also visible in our previous simulations of a small-mass dwarf galaxy (Siejkowski et al. 2014).

Our main goal was to study the consequences of the burst of star formation in the history of the galaxy. These effects have not been simulated before. In models v50x2, v50x5, and v100x2 we applied an increased SFR once in the galactic evolution. Slower rotators (v50x2 and v50x5) show rapid increase of the magnetic field as an immediate response to the increased SFR. However, after the initial growth, when the burst of star formation stops, the magnetic field value returns to its level corresponding to the unperturbed galactic evolution, as seen in Fig. 2. Total magnetic energy calculated in the whole simulation domain even falls below its "unperturbed" level, clearly showing the smaller growth rate (Fig. 1) over about 1 Gyr until the dynamo process comes to the same activity rate as before the burst of star formation. More violent starburst (model v50x5) events lead to a lower saturation level of the magnetic field value at the end of the simulated galactic history.

Our faster rotator (model v100x2) evolution does not show the initial increase of magnetic field as a response to the starburst event. The visible amplification rate, as well as its saturation level, are only slightly lower.

Star formation bursts frequently occur only in some parts of a galaxy (e.g. IC 10, LMC, NGC 4449). We tried to simulate such a situation by restricting the increased SFR to only one quarter of the galactic disk (models v50x1Q, v50x2Q, v50x5Q, 

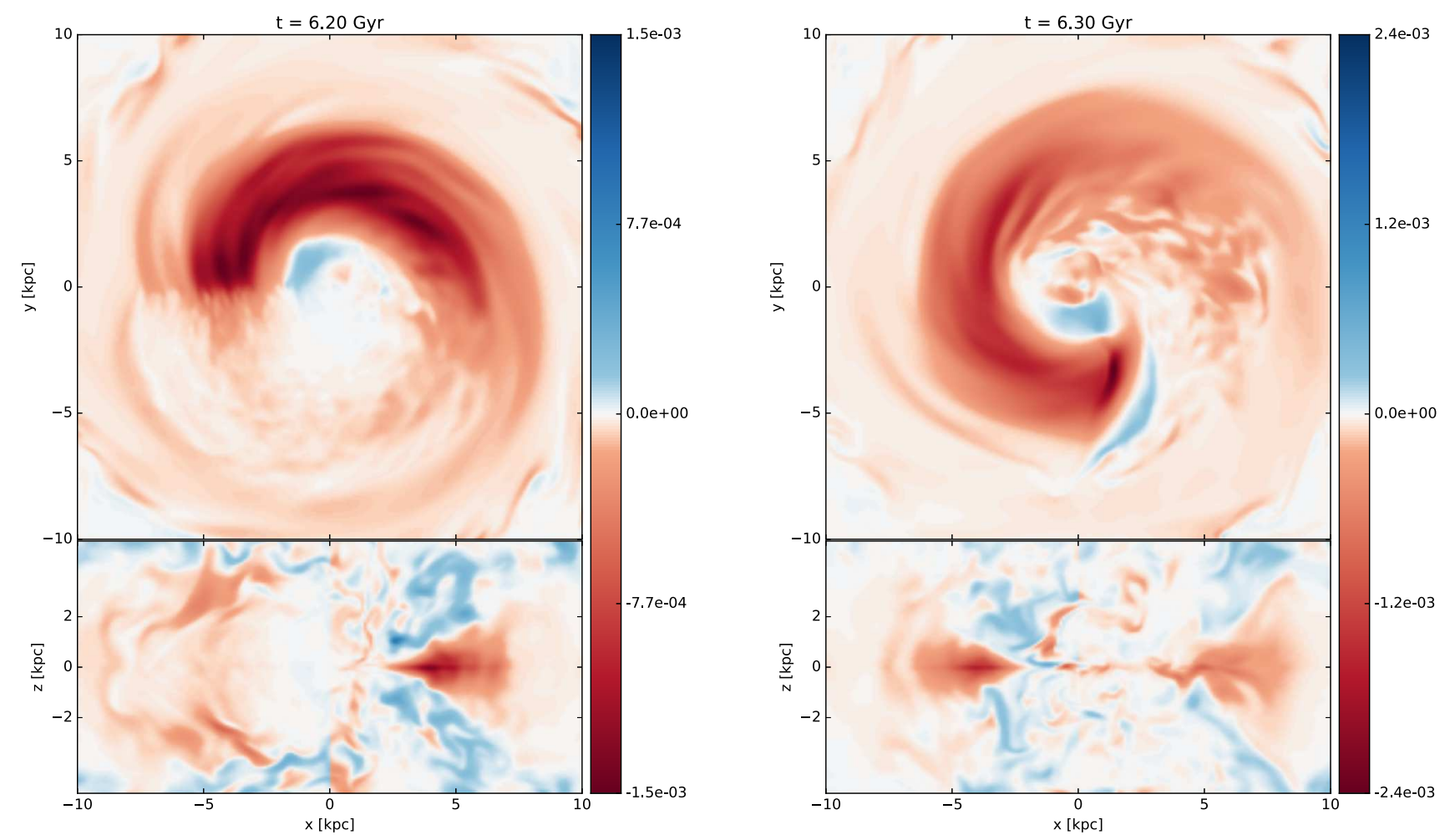

Fig. 4. Two snapshots of the azimuthal magnetic field component in the galactic disk and in the plane perpendicular to it. Left: at the beginning of a starburst event; right: at the end of the starburst. We note the change of the colour-scale in both plots.

v100x1Q, and v100x2Q). The magnetic field evolution caused by such localised starburst episodes is similar to in the corresponding "non-Q" models. All effects described above are present, but emphasised. This can be explained, knowing that while restricting the area for a burst of star formation, we kept the total number of SN explosions the same, that is, the local SFR in the burst area is four times higher.

It is interesting to see what is responsible for the growth of the magnetic field in the star formation burst event. Figure 4 shows the azimuthal magnetic field component in the galactic plane and in the plane perpendicular to it at the beginning (left panel) and at the end (right panel) of the burst event. Initially the increased SN activity disrupts the regularity of the magnetic field (its dominant azimuthal component). At the same time increased turbulence in the interstellar medium gives rise to more efficient dynamo action - most efficient at the boundary between areas of highly turbulent bursts and areas filled with more ordered magnetic field with no bursts. The galactic differential rotation propagates the boundary over the whole disk giving rise to global increase of the dynamo efficiency in a short time.

After the starburst event, the growth rate of the magnetic field decreases. We suggest that this is the consequence of a large fraction of the magnetic field expelled out of the galactic body together with the interstellar gas. The more violent the star formation involved is, the greater the volume of gas expelled from the galaxy (out of our computational domain), as shown in Fig. 5. Thus the dynamo efficiency can still remain at the same level. The apparent smaller magnetic field growth-rate is only a consequence of increased galactic wind carrying the magnetic field with it. A similar effect was observed in simulations of massive spirals by Chamandy et al. (2015) where stronger outflows in gaseous arms locally suppress the dynamo action and lead to the formation of inter-arm magnetic arms. The magnetic field growth rate returns to the same value, as only the gas escape rate returns to the level before the burst event. This never happens in the case of model v100x2Q where we observe an increased gas-escape rate until the end of the simulation.

Our simulations correspond to isolated galaxies. In actual galaxies, the periods of enhanced star formation could be triggered by tidal interactions with galaxy companions. This could induce in turn the gas infall (e.g. Nidever et al. 2013). The loss of gas by galactic wind could thus be supplemented with a new gas. Tracing how such processes would affect the evolution of the magnetic field in galaxies and change the above results requires new modelling.

From this work, we make the following conclusions. CRdriven dynamos can effectively amplify the magnetic field, even under dwarf/Magellanic-type galaxy conditions. The magnetic fields are amplified with $e$-folding time for the regular component of about $0.91 \mathrm{Gyr}$ and reach the saturation level after $12 \mathrm{Gyr}$ for models with $v_{\phi}=50 \mathrm{~km} \mathrm{~s}^{-1}$. For models where rotation is twice as fast, the $e$-folding is $0.44 \mathrm{Gyr}$, and the saturation is reached after 6 Gyr. The results are comparable to those reported by Hanasz et al. (2009b) and Kulpa-Dybeł et al. (2011), who studied CR-driven dynamos in more massive galaxies and find $e$ folding times for the regular component of the magnetic field of around 0.3 Gyr. The studies of energy balance in the galaxy show that the saturation point of the magnetic field growth is close to the equipartition level between the turbulent kinetic energy and the magnetic energy. Turbulent kinetic energy and CR energy are not in balance due to the value of the CR diffusion coefficient being lower than in reality (see e.g. Hanasz et al. 2009a).

We found that an episode of star-formation bursts increases the magnetic field, but for a short time only. It is followed by a smaller amplification rate until the dynamo action returns to the same efficiency rate as before the burst. Localised star formation bursts emphasise the effects of the global starburst event, that is, the momentary growth of magnetic field (localised to the 
H. Siejkowski et al.: Magnetic field evolution in dwarf and Magellanic-type galaxies
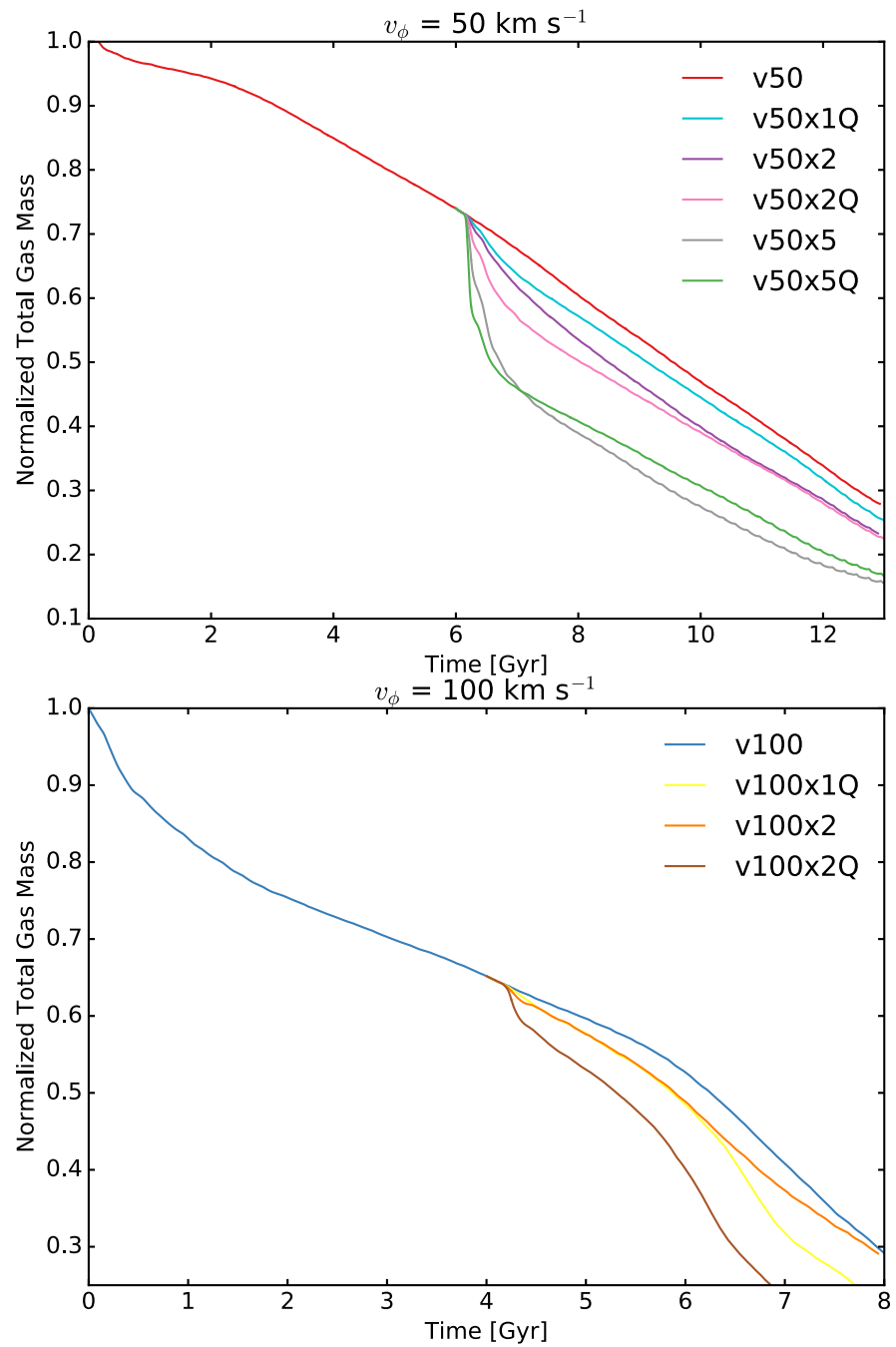

Fig. 5. Evolution of the total gas mass in a galaxy.

boundaries of a starburst), followed by smaller growth rate, and longer time needed for return of the dynamo action to its previous efficiency. We noticed that in larger galaxies, which are more massive and rotate faster, the effects of the starburst event were stronger. A substantial amount of gas is expelled from the dwarf/Magellanic-type galaxy (up to about $70 \%$ in $10 \mathrm{Gyr}$ ). The starburst event allows galactic gas to be expelled even faster.

Acknowledgements. We thank the anonymous referee for detailed and constructive comments. A substantial part of this work was supported by the Polish
National Science Centre through grant 2012/07/B/ST9/04404. This research was supported in part by PLGrid Infrastructure. The plots presented in this paper are rendered using Matplotlib (Hunter 2007).

\section{References}

Beck, R. 2016, A\&A Rev., 24, 4

Binney, J., \& Tremaine, S. 2008, in Galactic Dynamics: Second Edition, eds. J. Binney, \& S. Tremaine (Princeton, NJ: Princeton University Press)

Chamandy, L., Shukurov, A., \& Subramanian, K. 2015, MNRAS, 446, L6

Chyży, K. T., Beck, R., Kohle, S., Klein, U., \& Urbanik, M. 2000, A\&A, 355 128

Chyży, K. T., Weżgowiec, M., Beck, R., \& Bomans, D. J. 2011, A\&A, 529, A94

Chyży, K. T., Drzazga, R. T., Beck, R., et al. 2016, ApJ, 819, 39

Di Matteo, P., Bournaud, F., Martig, M., et al. 2008, A\&A, 492, 31

Dorfi, E. A. 2000, Ap\&SS, 272, 227

Giacalone, J., \& Jokipii, J. R. 1999, ApJ, 520, 204

Goedbloed, J. P. H., \& Poedts, S. 2004, in Principles of Magnetohydrodynamics, (Cambridge: Cambridge University Press), 129

Governato, F., Brook, C., Mayer, L., et al. 2010, Nature, 463, 203

Hanasz, M., Kowal, G., Otmianowska-Mazur, K., \& Lesch, H. 2004, ApJ, 605, L33

Hanasz, M., Otmianowska-Mazur, K., Kowal, G., \& Lesch, H. 2009a, A\&A, 498, 335

Hanasz, M., Wóltański, D., \& Kowalik, K. 2009b, ApJ, 706, L155

Hanasz, M., Lesch, H., Naab, T., et al. 2013, ApJ, 777, L38

Hunter, J. D. 2007, Comput. Sci. Eng., 9, 90

Jurusik, W., Drzazga, R., Jableka, M., et al. 2014, A\&A, 567, A134

Kennicutt, Jr., R. C. 1989, ApJ, 344, 685

Kepley, A. A., Mühle, S., Everett, J., et al. 2010, ApJ, 712, 536

Kowal, G., Lazarian, A., Vishniac, E. T., \& Otmianowska-Mazur, K. 2009, ApJ, 700,63

Kulpa-Dybeł, K., Otmianowska-Mazur, K., Kulesza-Żydzik, B., et al. 2011, ApJ, 733, L18

Mateo, M. L. 1998, ARA\&A, 36, 435

Mo, H., van den Bosch, F. C., \& White, S. 2010, Galaxy Formation and Evolution (Cambridge: CUP)

Nidever, D. L., Ashley, T., Slater, C. T., et al. 2013, ApJ, 779, L15

Ryu, D., Kim, J., Hong, S. S., \& Jones, T. W. 2003, ApJ, 589, 338

Schlickeiser, R. \& Lerche, I. 1985, A\&A, 151, 151

Schmidt, M. 1959, ApJ, 129, 243

Siejkowski, H., Soida, M., Otmianowska-Mazur, K., Hanasz, M., \& Bomans, D. J. 2010, A\&A, 510, A97

Siejkowski, H., Otmianowska-Mazur, K., Soida, M., Bomans, D. J., \& Hanasz, M. 2014, A\&A, 562, A136

Skillman, E. D., Hidalgo, S. L., Weisz, D. R., et al. 2014, ApJ, 786, 44

Snodin, A. P., Brandenburg, A., Mee, A. J., \& Shukurov, A. 2006, MNRAS, 373 643

Stepanov, R., Shukurov, A., Fletcher, A., et al. 2014, MNRAS, 437, 2201

Strong, A. W., Moskalenko, I. V., \& Ptuskin, V. S. 2007, Ann. Rev. Nucl. Particle Sci., 57, 285

Tolstoy, E., Hill, V., \& Tosi, M. 2009, ARA\&A, 47, 371

Wang, H.-H., Klessen, R. S., Dullemond, C. P., van den Bosch, F. C., \& Fuchs, B. 2010, MNRAS, 407, 705

Weisz, D. R., Dalcanton, J. J., Williams, B. F., et al. 2011, ApJ, 739, 5

Widrow, L. M. 2002, Rev. Mod. Phys., 74, 775

Woo, J., Courteau, S., \& Dekel, A. 2008, MNRAS, 390, 1453 